\title{
Enabling the Knowledge Management Environment in SEE Countries through Harmonized Codification Using Information and Communication Technologies
}

\author{
Msc. Napolon Beqiri \\ Doctoral Candidate \\ South-East European University, Macedonia
}

Doi:10.5901/ajis.2014.v3n2p301

Abstract

Considering the importance of creation of Knowledge Management Environment in SEE countries, comparable with the EU Environment, trends and situation in EU countries, and the variety of available Information and Communication Technology (ICT) Solutions on the market, it is a difficult and challenging task of deciding what type of ICT solutions to use in support of creation of Knowledge Management Environmentin SEE. This paper aims to research the state of harmonized codification using the Information and Communication Technologies in SEE countries by investigating the role of ICT in creation of the successful Environment for Knowledge Management. Codification of Knowledgeusing information and communication technology (ICT) have made it easier to acquire, store, or disseminate knowledge than ever before, many organizations are employing IT to facilitate sharing and integration of knowledge. SEE countries are being advised that to remain competitive, they must efficiently and effectively create, locate, capture, and share their knowledge and expertise, and have the ability to bring that knowledge to bear on problems and opportunities. SEE countries are showing a serious interest in implementing knowledge management processes and technologies, and are even beginning to adopt knowledge management as part of their overall development strategy. With the codification approach, more explicit and structured knowledge is codified and stored in knowledge bases. Examples of such IT tools are knowledge expert directories and video-conferencing tools. Both these Knowledge Management approaches are fundamental to understanding the importance of ICT in creation of Environment for Knowledge Management. Positive samples will be useful to be implemented within SEE countries, and as an impact, the contribution of the paper will be shown as a possible model of managing and motivating an environment that supports Knowledge Management Environment in SEE countries, through harmonized codification using Information and Communication Technologies.

Keywords: Knowledge, Management, Creativity, ICT, Codification, SEE, Environment

\section{Introduction}

While some view knowledge management as merely the current business fad, knowledge lies at the essence of humans as individuals. Respecting the role of knowledge and learning may be the most effective approach to building a solid and enduring competitive foundation for business organizations. Firms can derive significant benefits from consciously, actively and aggressively managing their explicit and explicable knowledge. Doing this in a coherent manner requires aligning the firm's organizational and technical resources and capabilities with its knowledge strategy. It requires mapping the firm's organizational and technical capabilities and constraints to its knowledge processing requirements. It may require significant organizational and technical interventions. The knowledge management architecture provides a framework for guiding this important effort.

Twenty-first century is called the era of fast changing world (Burke, 2000). The name is given to the era as a result of globalization effects, technologic developments, and the role of modern science in human life and demographic characteristics of different nations. In this regard, there is currently a compelling debate about the changing nature of business environments and the sources of competitiveness in advanced economies. It has been acknowledged that knowledge is rapidly overtaking capital and labor as the key economic resource in advanced economies. The tangible assets in an organization are widely recognized as vital elements in improving competitiveness. This has compelled academics and practitioners to discuss the way in which knowledge assets are managed; thus knowledge management is emerging as a significant concept in management science. KM has been extolled as "one of the major driving forces of organizational change and wealth creation" but remains a source, both theoretically and practically. Knowledge management has emerged as a discrete area in the study of organizations and is frequently cited as an antecedent of organizational performance. If organizations implement knowledge management practices successfully they are able to 
tackle the challenges thrown up by the knowledge society. Researchers have gone from studying the effects of ICT on economic-financial variables to studying its complementarities with intangible resources such as knowledge. But it is not clear how the relation between IT competency and knowledge management works. Empirical work in this area is lacking. Given that advances in information technology have made it easier to acquire, store, or disseminate knowledge than ever before, many organizations are employing IT to facilitate sharing and integration of knowledge. But considering the complexity of KM initiatives and the variety of IT solutions available on the market, executives must often confront the challenging task of deciding what type of IT solutions to deploy in support of their initiatives.

\section{Basic Approaches to Knowledge Management with IT support}

There are two basic approaches to Knowledge Management for which IT can provide support: codification and personalization.

With the codification approach, more explicit and structured knowledge is codified and stored in knowledge bases. The main role of IT here is to help people share knowledge through common storage so as to achieve economic reuse of knowledge. An example of such IT tools is electronic knowledge repositories. With the personalization approach, more tacit and unstructured knowledge is shared largely through direct personal communication.

The main role of IT here is to help people locate each other and communicate so as to achieve complex knowledge transfer. Examples of such IT tools are knowledge expert directories and video-conferencing tools. Both these KM approaches are fundamental to understanding the role of IT in KM. In addition, IT facilitates the process of knowledge transfer. Technology enables individuals to coordinate the logistics of face to face meetings. It can also be used to catalogue expertise of organizational members and a result facilitating access to the right people and enhancing knowledge sharing. Certain systems (e.g., groupware or collaborative systems) provide a virtual space where the participants can process the information and knowledge in real time, giving them more chance to interact (Roger $C$. Palmer, 2004). Exchange spaces become the ideal place to develop innovative and creative behaviors around problems and situations. One of the most important characteristics of these exchange spaces and virtual communities is that they are founded on the democratization of knowledge, so they enable the appearance of natural flows of transference and collaboration and consequently favour creativity and innovation.

On other hand, IT supports the process of knowledge codification and storage. IT facilitates the standardization of certain Knowledge management necessary mechanisms to codify and store knowledge.

In order to be useful, however, knowledge stores must be accessible to firm members and must be in a form that will enable each member to interpret in a similar manner, therefore becoming a part of the whole firm's knowledge base. IT, with its protocols and platform standards, provides an ideal mechanism for connecting widely dispersed individuals via a common system and enabling firm members to access more easily the knowledge that is stored in memory, so that new information can be interpreted and synthesized with existing knowledge,to perform intelligently and to sustain their competitive advantage by developing their knowledge assets. So, it is essential to know how to generate knowledge, how to disseminate it in the organization and what factors facilitate these processes.

In recent years, several researchers have associated knowledge management with the development of information and communication technologies. The new technologies are characterized by their capacity to influence the traditional ways of understanding certain organizational phenomena and behaviors and affect how organizations deal.

\section{Perspectives of Knowledge Management in Theory and Practice}

A wide range of study on the Knowledge Management exists and is being explored as an ongoing academic discipline; approaches vary by author and school. As this discipline matures, academic debates have increased regarding both the theory and practice of Knowledge Management, to include the following perspectives:

- Techno-centric with a focus on technology, ideally those that enhance knowledge sharing and creation Organizational with a focus on how an organization can be designed to facilitate knowledge processes best.

- Ecological, with a focus on the interaction of people, identity, knowledge, and environment.

Regardless of the school of thought, core components of Knowledge Managment include people, processes, technology, culture, structure, depending on the specific perspective. Different schools of thought Knowledge Management include different studies through which KM can be viewed and explained.

Technology context addresses the existing information technology infrastructure and abilities supporting the knowledge management architecture. While it is considered that knowledge management is 10\% technology and $90 \%$ 
people, without the ability to collect, index, store, and distribute explicit knowledge electronically to where and when it is needed, the organizational capabilities and priority will not be fully exploited. However, the technology doesn't need to be complex or edge to provide significant benefit. Its absence, however, would have prevented from effectively managing their knowledge.

New software technologies for personal productivity, research documentation and workgroup applications are analysed as instances of knowledge "codification". The analysis reveals that the social processes governing disclosure and cooperation in codification processes are as necessary as technological capabilities for some of the most promising applications

In recent years, several researchers have associated knowledge management with the development of information and communication technologies, (ICT). The new technologies are characterized by their capacity to influence the traditional ways of understanding certain organizational phenomena and behaviours and affect how organising should be done more effectively.

Researchers have gone from studying the effects of ICT on economic-financial variables to studying its complementarities with tangible resources such as knowledge. Given that advances in information technology (IT) have made it easier to acquire, store, or disseminate knowledge than ever before, many organizations are employing IT to facilitate sharing and integration of knowledge. But considering the complexity of KM initiatives and the variety of IT solutions available on the market, executives must often confront the challenging task of deciding what type of IT solutions to deploy in support of their KM initiatives.

\section{The Role of IT in Knowledge Management}

Knowledge Management approaches are fundamental to understanding the role of IT in KM. In addition, IT facilitates the process of knowledge transfer. Technology enables individuals to coordinate the logistics of face to face meetings. It can also be used to catalogue expertise of organizational members and a result facilitating access to the right people and enhancing knowledge sharing. Certain systems (e.g., groupware or collaborative systems) provide a virtual space where the participants can process the information and knowledge in real time, giving them more chance to interact. Exchange spaces become the ideal place to develop innovative and creativebehaviours around problems and situations. One of the most important characteristics of these exchange spaces and virtual communities is that they are founded on the democratization of knowledge, so they enable the appearance of natural flows of transference and collaboration and consequently favour creativity and innovation. On other hand, IT supports the process of knowledge codification and storage. IT facilitates the standardization and automation of certain tasks, supporting the transformation of tacit knowledge into explicit knowledge (Beqiri, 2011). Similarly, IT also provides the necessary mechanisms to codify and store knowledge. In order to be useful, however, knowledge stores must be accessible to firm members and must be in a form that will enable each member to interpret in a similar manner, thereby becoming a part of the whole firm's knowledge base. IT, with its protocols and platform standards, provides an ideal mechanism for connecting widely dispersed individuals via a common system and enabling firm members to access more easily the knowledge that is stored in memory bins, so that new information can be interpreted and synthesized with existing knowledge.

\section{Case Study}

Raising the level of information technology use in supply, wholesale and retail sales, inventory, analysis and reporting of sales channel items (products), requires rapid recognition and implementation of current technologies to identify trade items. Technologies that are used today to identify trade items are Barcode and RFID technology (Radio Frequency Identification Data).

In South East Europe (SEE) Barcode identification of items through the expansion of common market is happening thanks to the application of new technologies. This technology requires that retail trade items (products) will contain a unique identification number (expressed graphically with bar code).Bar code generated on a regular basis, determined and well printed, enables your items to go through the sales channel without problems, commitments and additional costs.

Since it was discovered, barcode, in the 20th century, have become indispensable part of modern civilization. Their use is very broad, and the technology of barcodes is constantly growing. Most people today have seen barcodes. They are printed on almost every item in the store to buy food. There are many types of barcode (about 300, but the most common that we see are UPC or EAN (Palmer, 2004). Another code, quite famous is Code 39 (also called Code 3 and 9). 


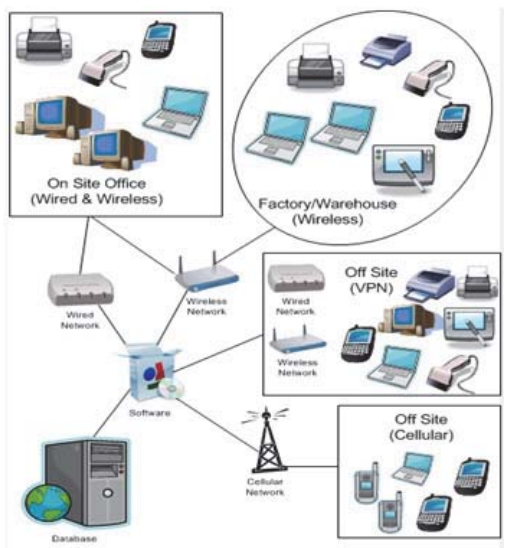

Figure 1. The possibility of bar coding in many countries and in many networks such as an office, factory and warehouse, whether wire bound or wireless storage of data on some devices.

\section{Bar Code Application in SEE Countries}

Some modern barcode applications include:

- Practically every item purchased from a grocery store, other stores and any other commercial commodity hasa printed barcode. This helps a lot in maintaining records for a large number of items in the store and also reduces requirements of shop manager (while traders cannot mix the prices of cheap items they valued more expensive). So barcode application both with consumers and retailers have benefited from the savings it has achieved.

- documentation management tools often allow you to identify the barcode to facilitate the separation and indexing of documents as they are scanned and electronically stored data for those documents .

- Monitoring and keeping records for the movement of goods, including cars with rent, airline baggage, mail , plane tickets and packaged items .

- Scientist have established small barcodes even on each individual bees to track the habits and behaviour of insects .

- Many tickets now have barcodes that must be evaluated for validity before being allowed an entry to ticket holders in the field of sport, cinema, theatre, airport, transport etc. .

- When barcodes are used at vehicles they can be placed in front or back of the vehicle.

\section{The Secret Coding Pillars}

EAN Coding is the one commonly used in EU. EAN coding presentation by pillar coding is done in two blocks: the left block which contains digits 2 to 7 and right block contains the digits 8 to 13. The first figure is hidden by a particular method on the left block. Two columns of money in the beginning and end of the coding are needed as boundary pillars or sides, and in between a separator bar.

The figures used for encoding differ in coding tables. On the right half of the coding is used only the left half of $C$ coding and is not so easily to be coded by the first digit EAN must be processed as follows : Depending on the first digit EAN coding, half of the left coded by coding samples A or B. A and B are different from each other, and for this reason code can be read from different directions. This complicated coding has been necessary in order that the reader coding pillars can also refine UPC coding that is used in the U.S.

For encoding, the colors and size columns are almost unconditional. This means that the tools for reading these codes are exposed to high demand. These tools are called scanners and work with a precision laser beam which slides over the EAN code. These codes are possible to identify even moving aroundand by a large distance (Beqiri, 2011).

The laser sends a beam through a curved polygonal mirror 50 times a second on the field. Different reflections of the laser beam, by columns of black and white, delivered through a system of mirrors and a lens to image sensor where 
the impulses then change in to reading.

Systems of data collection through barcodes offer enormous benefits for each type of business. By providing a solution for data collection through barcode, data recording is faster and more accurate, lower costs, errors are minimized and inventory management is much easier. Data entry via keyboard creates an average of one in 300 printing error. Data entry through the barcode has a likelihood of error of one in three million (Burke, 2005).

\section{Reducing Expenses with the Codification of Products and Goods}

The biggest benefit of collecting data through barcode are reasonable expenses. In many cases, the cost savings justify the cost of electronic system for data collection. But this is not the only benefit to managers.

Reducing the loss of revenue from errors in data collection is much higher than traditional ways. We know that if we have a significant error in the documents than that will start a chain reaction of expenses in time, finance etc.

Levels of inventory: use of barcodes is one of the best ways to reduce both inventory levels and capital expenditure savings. Maintaining strong hand in inventory can save us a considerable amount of money

\section{Improvement of Management through Codification}

Management through codification makes posible Improvement and better decision-making. Although it is difficult to measure, however, improved management is an important benefit. It improves technology management because automatic data collection may be the biggest benefit of bar coding system. Remember that easily collected barcode system can be difficult or impossible to meet through other means. This enables managers to make decisions based on more accurate data that can influence the direction that will take the department or company.

Another benefit is in faster access to information. This benefit is similar to better decision making. Having possessed the most accurate information puts us in lead with our competitors.

\section{Samples of Standardized Codes in SEE Countries}

The UPS number -codes for Regional and SEE countries with some specific codes or products are as follows:

- 020 - 029 Restricted distribution

- 040 - 049 Restricted distribution (MO defined)

- $050-059$ coupons

- $200-299$ Restricted distribution

- 380 Bulgaria

- 383 Slovenia

- 385 Croatia

- 387 Bosnia and Herzegovina

- 389 Montenegro

- 530 Albania

- 531 Macedonia

- 860 Serbia

- 950 GS1 Global Office: Special applications

- 951 EPCglobal: Special applications

- 960 - 969 GS1 Global Office: GTIN-8 allocations

- 977 Serial publications (ISSN)

The ISBN codes used for some counries of SEE are

- 9951 Kosovo

- 99927 Albania

Implementing bar-codes in a regular manner makes it easier to trace the products and goods even when moving within the region of SEE, hence giving more time to mangers to cooperate and share KM. 


\section{Conclusion}

The codification approach to KM is often implemented to facilitate economic reuse of knowledge. Knowledge is codified, stored electronically, and made available to employees via common technological platforms throughout the entire organization. For instance, Ernst and Young have developed the Centre for Business Knowledge as a central repository holding its 40 areas of operational knowledge (Sarvary 1999). Similarly, KWorld of KMPG and SAP R/3 Knowledge Library of Siemens Business Services (Davenport and Probst 2000) are valuable knowledge resource repositories for their employees. These organizations deliver their knowledge resources to employees through common technological platforms such as Microsoft Office, Lotus Notes, and web browsers.

In contrast, knowledge needed by sales teams (e.g., market characteristics, product capabilities, and service tips) can be more readily codified and thereby cost effectively disseminated to sales teams. Thus, the codification strategy to KM can be employed for such functions. For example, Hewlett Packard uses its Electronic Sales Partners as a knowledge base of technical product details, sales and marketing tactics, and customer account history for its sales force (Teigland, 2010). Xerox deploys the Eureka knowledge repository to provide its service engineers with access to technical tips for servicing photocopier machines (Hickins, 1999). In Microsoft, the Internal Technical Education knowledge repository (comprising online learning, live class schedules, and white papers) provides knowledge and training to its field sales force (Burke, 2005).We can conclude that importance of barcode is high because product with barcode included is known in all the world and also barcode make easy to work and read information about that product.

During these 30 years barcode and identification system has changed behavior and benefits costumers all around the world as well as in SEE Countries. The results of the paper shows that Barcode systems provides an array of benefits, including operational efficiency, better customer service, and improved visibility of key business information to management.

The findings of this paper concludes advanteges of codification through Bar coding in SEE Countries which shows beter results in: Speed, Accuracy, Ease of Implementation and cost effectivness, such as follows:

- Speed: A bar code label of twelve characters can be winded in approximately the time it takes a keyboard operator to make two keystrokes.

- Accuracy: For every 1,000 characters typed by a keyboard operator, there are an average of ten keying errors. For an Optical Character Reader (OCR), there is one error in every 10,000 reads.

- Ease of Implementation: Operators of bar code scanners can learn to use the equipment effectively in less than 15 minutes.

- Cost Effectiveness: Barcode systems create value not only by saving time, but also by preventing costly errors.

Nowadays, goods and services cannot be imagined without barcode incorporation. So with all rights we can declare that products or goods can't exist without having incorporated barcode with all important information about those products or goods, barcodes are making simple automated system for reading product data, bringing to us efficiency and accuracy in wholesale, retail, import-export in SEE countries and worldwide and they play important role in international trade.

\section{References}

Harry E. Burke (2005), Automating Management Information Systems: Principles of Barcode Applications, Thomson Learning, ISBN 0442-20667-4

Harry E. Burke, (2000) Handbook of Bar Coding Systems, Van Nostrand Reinhold Company, ISBN 978-0-442-21430-2, 219 pages

Hickins M. (1999), "Xerox shares it's knowledge", Management Review, Vol-88, No. 9, pp.40-5.

Teigland R. (2010), Born virtuals and Avapreneurship: A case study of achieving succesful outcomes in Peace train-A second life organization", Jounal of Virtual World Research, Special issue on virtual Economies, Virtual goods and Service Delivering in Virtual World.

Beqiri, Edmond (2011), Ekonomia dixhitale dhe Biznesi elektronik, European College Dukagjini, Dukagjini

Roger C. Palmer, 2004, The Bar Code Book, Helmers Publishing, ISBN 0-911261-09-5, 386 pages

\section{Other sources}

http://www.idautomation.com/sitemap/barcode http://www.barcode.com/category/applications/ http://www.barcodesymbols.com/information/barcodes.htm http://www.makebarcode.com/info/intro.html http://www.barcoding.com/information/learn_about_barcodes.shtml 